> La maladie de Fabry (MF) est une pathologie héréditaire du métabolisme des glycosphingolipides resultant du déficit en $\alpha$-galactosidase $A$, une enzyme lysosomale. Le déficit enzymatique conduit à l'accumulation de glycosphingolipides dans le plasma et les lysosomes de nombreux types cellulaires. Bien que la maladie de Fabry soit un modèle de maladie multi-systémique, aucune donnée n'est disponible concernant l'atteinte osseuse. Dans ce travail, une ostéodensitométrie fut mesurée en deux sites, la colonne vertébrale lombaire et la tête fémorale chez 23 hommes hémizygotes, âgés en moyenne de 31 ans et atteints de la forme classique de la maladie de Fabry. Selon la classification OMS de la perte de densité minérale osseuse, 20 des 23 patients ( $88 \%$ ) présentaient soit une ostéopénie $(n=11)$ soit une ostéoporose ( $n=9)$ à l'un et/ou l'autre site. <

\section{Atteinte osseuse de la maladie de Fabry}

Dominique P. Germain, Karelle Benistan, Lilit Khatchikian, Céline Mutschler

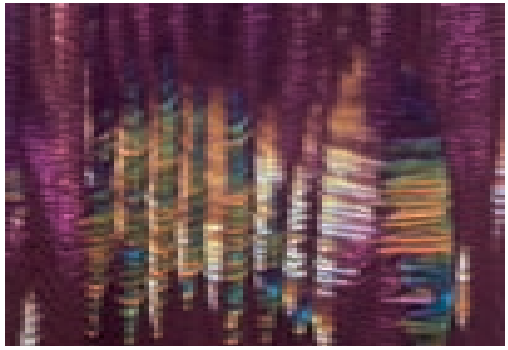

D.P. Germain, K. Benistan,

L. Khatchikian : Unité de Génétique Clinique. C. Mutschler: Service de Radiologie. Hôpital Européen Georges Pompidou, 20, rue Leblanc, 75015 Paris, France

dominique.germain@egp.aphp.fr

Les résultats d'ostéodensitométrie furent exprimés en utilisant le T-score et le Z-score pour tous les patients. Selon la définition OMS de la perte de densité minérale osseuse, celle-ci fut qualifiée de normale en cas de valeur du T-score déviant de moins d'un écart type; l'ostéopénie fut définie par un T-score compris entre - 1 et - 2,5 déviation standard et l'ostéoporose par un T-score inférieur à - 2,5 déviation standard; la survenue d'une fracture de fatigue, combinée à ce dernier T-score, définissant l'ostéoporose sévère [3].

\section{Résultats}

Seuls trois patients sur $23(11 \%)$ avaient un T-score normal aux deux sites étudiés, les 20 patients restant ayant au minimum une ostéopénie à l'un des deux sites étudiés [4]. Les données d'ostéodensitométrie sont détaillées dans le Tableau I.

\section{Discussion}

Vingt trois patients, de sexe masculin, avec un diagnostic de maladie de Fabry confirmé par enzymologie et/ou génétique moléculaire furent inclus dans cette étude. Une ostéodensitométrie osseuse, avec étude de deux sites, la colonne lombaire basse et la tête fémorale, fut systématiquement réalisée pour chacun des patients avant thérapie enzymatique substitutive $(n=14)$, ou dans les six premiers mois suivant son initiation $(n=9)$. Une étude du métabolisme phosphocalcique, du métabolisme du fer, un bilan thyroïdien et parathyroïdien furent également réalisés.
Un certain nombre d'étiologies peuvent a priori être exclues pour expliquer les résultats obtenus :

- la transplantation rénale, puisqu'aucun patient de cette série n'était greffé ;

- l'insuffisance rénale chronique: seuls cinq malades sur 23 étaient en insuffisance rénale chronique. $0 r$, parmi les 18 autres patients, la majorité présentait également une ostéopénie ou une ostéoporose ;

- la ménopause, puisque la population étudiée correspondait uniquement à des hommes jeunes ; 
- la corticothérapie et les radiations ionisantes, deux facteurs bien connus pour favoriser la survenue d'une ostéopénie mais auxquels aucun patient n'avait été exposé ;

- le tabagisme, qui était peu important dans cette cohorte de patients;

- la réduction importante d'activité physique qui est un facteur que l'on se devait de considérer dans le cas de la maladie de Fabry. Toutefois, dans cette cohorte, seuls trois malades ont admis avoir une réduction d'activité physique, tandis que les 20 malades restant avaient une activité physique et sociale normale.

Parmi les étiologies qu'il nous semble falloir privilégier :

- la malnutrition; un indice de masse corporelle réduit est souvent retrouvé chez les patients atteints de maladie de Fabry;

- une malabsorption avec, en conséquence, une diminution de l'absorption intestinale de la vitamine D. La maladie cœliaque est d'ailleurs un diagnostic différentiel de la maladie de Fabry dans l'enfance et l'association entre maladie cœliaque et ostéoporose est aujourd'hui connue [5] ;

- les douleurs chroniques et la dépression; de nombreux articles dans la littérature mettent en évidence l'existence d'une ostéopénie, voire d'une ostéoporose, chez des malades douloureux chroniques ou déprimés chroniques;

- l'utilisation de la carbamazépine, pour le traitement des douleurs neuropathiques de la maladie de Fabry, qui perturbe le métabolisme phosphocalcique [6] ; - des dépôts de Gb3 au niveau du système vasculaire osseux, qui pourraient conduire à un appauvrissement de la nutrition des cellules et de la masse minérale. Des données histomorphométriques permettraient probablement de mieux préciser la nature d'un éventuel découplage des fonctions ostéoclasiques et ostéoblastiques. L'accès à des biopsies osseuses serait donc précieux mais est extrêmement rare dans le cas de la maladie de Fabry [7].

\section{Conclusion}

La fréquence précédemment insoupçonnée de l'ostéopénie et de l'ostéoporose a été pour la première fois démontrée dans ce travail. Aucune traduction clinique n'a pour l'instant été rapportée de ce que nous venons de découvrir ici. En effet, le nombre de patients atteints de maladie de Fabry, parmi les milliers de cas rapportés dans la littérature, ayant présenté d'authentiques fractures, est extrêmement limité. L'analyse du registre international de la maladie de Fabry (Fabry Registry ${ }^{\circledR}$, www.fabryregistry.com) qui compte

\begin{tabular}{|ll|}
\hline Classes OMS & Nombre de patients (\%) \\
\hline Normal & $3(13 \%)$ \\
\hline $\begin{array}{l}\text { Ostéopénie à un site, } \\
\text { valeur normale à l'autre }\end{array}$ & $8(35 \%)$ \\
\hline Ostéopénie aux deux sites & $3(13 \%)$ \\
\hline Ostéoporose à un site, valeur normale à l'autre & $1(4 \%)$ \\
\hline Ostéoporose à un site, ostéopénie à l'autre & $6(26 \%)$ \\
\hline Ostéoporose aux deux sites & $2(9 \%)$ \\
\hline Ostéoporose sévère & Aucun \\
\hline
\end{tabular}

Tableau I. Résultats des ostéodensitométries de la colonne vertébrale basse et de la tête fémorale exprimés en T-scores (OMS : Organisation Mondiale de la Santé). désormais plus d'un millier de patients à travers le monde, pourra être mise à profit pour étudier la prévalence exacte des cas de fractures pathologiques.

Si la thérapie enzymatique substitutive désormais disponible entraîne un allongement de l'espérance de vie des patients, il pourrait être important de prévenir la survenue de fractures de fatigue chez des sujets plus âgés au moyen de thérapeutiques adjuvantes comme les diphosphonates, à moins que l'enzymothérapie ne parvienne à elle seule à prévenir, si elle est instaurée suffisamment tôt, la perte de densité minérale osseuse. $\diamond$

\section{SUMMARY}

Bone involvement in Fabry disease

Fabry disease (FD) is an X-linked inborn error of metabolism resulting from the deficiency of the lysosomal hydrolase, $\alpha$-galactosidase $A$. The enzymatic defect leads to the widespread deposition of glycosphingolipids in the plasma and lysosomes of various cell types resulting in cellular, tissue, and organ dysfunction. While the classic form of FD is known to be a multi-system disorder, no data are available regarding involvement of bones. In this study, bone mineral density of the lumbar spine and the femoral neck was assessed by dual-energy X-ray absorptiometry (DEXA) in 23 hemizygous male patients with a mean age of 31 years (16-60 years) affected with classic FD. Using the World Health Organization classification, 20 of the 23 patients $(88 \%)$ with FD had either osteopenia $(n=11)$ or osteoporosis $(n=9)$ at one or both sites. $\diamond$

\section{RÉFÉRENCES}

1. Rigante D, Caradonna P. Secondary skeletal involvement in Sanfilippo syndrome. QJM $2004 ; 97: 205-9$.

2. Lebel $\varepsilon$, Dweck A, Foldes AJ, et al. Bone density changes with enzyme therapy for Gaucher disease. J Bone Miner Metab 2004 ; 22 : 597-601.

3. Kanis JA. Assessment of fracture risk and its application to screening for postmenopausal osteoporosis : synopsis of a WHO report. WHO Study Group. Osteoporos Int $1994 ; 4: 368-81$.

4. Germain DP, Benistan K, Boutouyrie P, et al. Osteopenia and osteoporosis: previously unrecognized symptoms of Fabry disease. Clin Genet 2005 ; 68 : 93-5.

5. Cellier C, Flobert C, Cormier C, et al. Severe osteopenia in symptom-free adults with a childhood diagnosis of coeliac disease. Lancet 2000; $355: 806$.

6. Farhat $G$, Yamout $B$, Mikati MA, et al. Effect of antiepileptic drugs on bone density in ambulatory patients. Neurology $2002 ; 58: 1348-53$.

7. Horiuchi H, Saito N, Kobayashi S, et al. Avascular necrosis of the femoral head in a patient with Fabry's disease : identification of ceramide trihexoside in the bone by delayed-extraction matrix-assisted laser desorption ionization-timeof-flight mass spectrometry. Arthritis Rheum $2002 ; 46$ : 1922-5. 